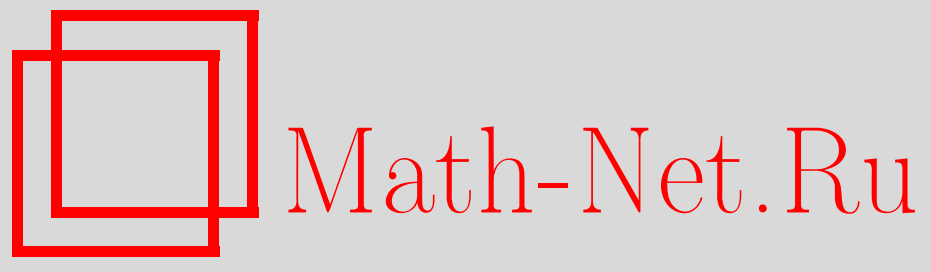

В. И. Скалыга, Условия точности в многомерных аналогах неравенства В. А. Маркова, Матем. заметки, 2006, том 80, выпуск 6, 950-953

DOI: https://doi.org/10.4213/mzm3373

Использование Общероссийского математического портала Math-Net.Ru подразумевает, что вы прочитали и согласны с пользовательским соглашением http://www . mathnet.ru/rus/agreement

Параметры загрузки:

IP: 18.234 .197 .8

26 апреля 2023 г., $14: 46: 16$

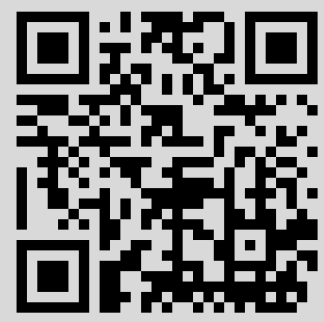




\section{УСЛОВИЯ ТОЧНОСТИ В МНОГОМЕРНЫХ АНАЛОГАХ НЕРАВЕНСТВА В. А. МАРКОВА}

\section{В. И. Скалыга}

Введение. Пусть $X$ - нормированное векторное пространство над $\mathbb{R}$. Через $\mathfrak{P}_{n}(X, \mathbb{R})$ обозначим пространство всех полиномов степени не выше $n$, отображающих $X$ в $\mathbb{R}$, т.е. множество всех функционалов вида

$$
P_{n}(x)=\sum_{j=0}^{n} u_{j}(x, \ldots, x), \quad x \in X,
$$

где $u_{j}: X^{j} \rightarrow \mathbb{R}-j$-линейный непрерывный функционал. Значение $k$-й производной Фреше полинома $P_{n}$ в точке $x$ на кортеже векторов $\left(h_{1}, \ldots, h_{k}\right)$ будем обозначать как $P_{n}^{(k)}(x)\left[h_{1}, \ldots, h_{k}\right]$. Если $h_{1}=\cdots=h_{s}=h$, то

$$
P_{n}^{(k)}(x)\left[h_{1}, \ldots, h_{k}\right]=P_{n}^{(k)}(x)\left[h^{s}, h_{s+1}, \ldots, h_{k}\right] .
$$

Пусть $\mathscr{L}^{k}=\mathscr{L}^{k}(X, \mathbb{R})$ пространство ограниченных симметричных $k$-линейных функционалов на $X^{k}$ и

$$
\left\|P_{n}^{(k)}(x)\right\|_{\mathscr{L}^{k}}=\sup _{\left\|h_{i}\right\|=1,1 \leqslant i \leqslant k}\left|P_{n}^{(k)}(x)\left[h_{1}, \ldots, h_{k}\right]\right| .
$$

В настоящей работе $K$ - выпуклое ограниченное замкнутое тело, симметричное относительно $\theta_{X}$ - нуля пространства $X, B=\{x \in X:\|x\| \leqslant 1\}$. Для ограниченного множества $G \subset X$ положим

$$
\left\|P_{n}\right\|_{G}=\sup _{x \in G}\left|P_{n}(x)\right|, \quad\left\|P_{n}^{(k)}\right\|_{G}=\sup _{x \in G}\left\|P_{n}^{(k)}(x)\right\|_{\mathscr{L}^{k}} .
$$

Мы будем использовать следующие характеристики тела $K$ :

- $\rho(\cdot)$ - функция Минковского для тела $K, r_{h}=\|h\| / \rho(h), r(K)=\inf _{\|h\|=1} r_{h}$;

- $\rho_{1}(x, k)=\max \left\{\rho(x), \cos \left(\{k\}_{2} \pi /(2 n)\right)\right\}$, где $\{k\}_{2}$ - остаток от деления $k$ на 2 .

В работе [1] автором получены следующие неравенства для $P_{n} \in \mathfrak{P}_{n}(X, \mathbb{R})$ и $\left\|P_{n}\right\|_{K} \leqslant 1$ :

$$
\begin{aligned}
\left|P_{n}^{(k)}(x)\left[h^{k}\right]\right| & \leqslant \frac{T_{n}^{(k)}(1)}{r_{h}^{k}}, \quad x \in K \subset X, \\
\left|P_{n}^{(k+s)}(x)\left[h^{k}, \bar{x}^{s}\right]\right| & \leqslant \frac{T_{n}^{(k+s)}(\rho(x))}{r_{h}^{k} r_{\bar{x}}^{s}}, \quad \rho(x) \geqslant 1, \\
\left|P_{n}^{(k)}(x)\left[h_{1}, \ldots, h_{k}\right]\right| & \leqslant \frac{c_{k} T_{n}^{(k)}\left(\rho_{1}(x, 2)\right)}{r^{k}(K)},
\end{aligned}
$$

где $T_{n}(\cdot)$ - полином Чебышева, $T_{n}(t)=\cos (n \arccos t),|t| \leqslant 1,\|h\|=\left\|h_{i}\right\|=1, i=1, \ldots, k$, $\bar{x}=x /\|x\|, 1 \leqslant c_{k} \leqslant k^{k} / k$ ! и, если $X-$ предгильбертово пространство, то $c_{k}=1$. В настоящей работе будут установлены необходимые и достаточные условия для выполнения

Работа выполнена при финансовой поддержке Российского фонда фундаментальных исследований, грант № 05-01-00275.

(C) В.И. СКАЛЫгА, 2006 
равенства в (1), (2) и выполнения равенства в (3) для $m$-мерного евклидового пространства $E^{m}$. Если $X=\mathbb{R}$, то неравенство (1) является известным [2] неравенством Маркова. Для $X=\mathbb{R}$ Шеффер и Даффин [3] получили при $\left\|P_{n}\right\|_{[-1,1]} \leqslant 1$ оценку

$$
\left|P_{n}^{(k)}(t)\right| \leqslant N_{n, k}(t)= \begin{cases}M_{n, k}(t)=\left|T_{n}^{(k)}(t)+i S_{n}^{(k)}(t)\right|, & |t| \leqslant \xi_{1}^{(k)}, \\ \left|T_{n}^{(k)}(t)\right|, & |t| \geqslant \xi_{1}^{(k)},\end{cases}
$$

где $S_{n}(t)=\sin (n \arccos t)=n^{-1}\left(1-t^{2}\right)^{1 / 2} T_{n}^{\prime}(t), \xi_{1}^{(k)}$ - максимальный нуль функции $S_{n}^{(k)}(\cdot)$ на $[-1,1], i^{2}=-1$. Функция $N_{n, k}(\cdot)$ возрастает на $[0, \infty]$. Равенство в $(4)$ достигается только на полиномах $\pm T_{n}(\cdot)$ в $n-k+1$ нулях функции $S_{n}^{(k)}(\cdot)$ и при $|t| \geqslant \xi_{1}^{(k)}$.

В [4] и [5] автором получены представление для $M_{n, k}^{2}(t)$

$$
M_{n, k}^{2}(t)=\sum_{j=0}^{k-1} a_{j}^{(k)}(n)\left(1-t^{2}\right)^{-(k+j)}, \quad a_{j}^{(k)}(n)>0, \quad j=0, \ldots, k-1,
$$

и рекуррентные формулы (по $k$ ) для вычисления $a_{j}^{(k)}(n)$. В работе [6] доказано равенство

$$
a_{j}^{(k)}(n)=n^{2} C_{k-1+j}^{2 j} \prod_{i=1}^{j}(2 i-1)^{2} \prod_{\alpha=j+1}^{k-1}\left(n^{2}-\alpha^{2}\right) .
$$

В работах автора [4] и [5] для $K \subset X,\left\|P_{n}\right\|_{K} \leqslant 1$ установлено неравенство

$$
\left|P_{n}^{(k+s)}(x)\left[h_{1}, \ldots, h_{k}, \bar{x}^{s}\right]\right| \leqslant \frac{\alpha_{k} N_{n, k+s}(\rho(x))}{r_{h_{1}} \cdots r_{h_{k}} r_{\bar{x}}^{s}},
$$

где $\alpha_{k}=k^{k / 2},\left\|h_{i}\right\|=1, i=1, \ldots, k$. Если $k=1$ и $\rho(x)$ является нулем функции $S_{n}^{(1+s)}(\cdot)$ либо $\rho(x) \geqslant \xi_{1}^{(1+s)}$, то существуют $P_{n}$ и $h_{1}$, для которых в $(7)$ имеет место равенство. При $k=1$ и $s=0$ оценку (7) установил Сарантопулос [7]. Для $K=B \subset E^{m}, k=1, s=0$ неравенство (7) доказал Келлог [8]. Если $K$ - тело из $E^{m}$, ограниченное эллипсоидом и $h_{1}=\cdots=h_{k}=h$, то $\alpha_{k}=1$ [4], [5]. Позднее для евклидового шара это получили Муньос и Сарантопулос [6].

Формулировки результатов. Пусть $x, h \in X, K \subset X,\|h\|=1, y \in \operatorname{span}\{h, x\}$ и $y=t_{1} r_{x} \bar{x}+t_{2} r_{h} h, A_{0}=\left\{y:\left|t_{1}\right|+\left|t_{2}\right| \leqslant 1\right\}, A_{1}=\left\{y:\left|t_{1}+t_{2}\right| \leqslant 1\right\}, A_{2}=\left\{y:\left|t_{1}-t_{2}\right| \leqslant 1\right\}$, $K_{x h}=K \cap \operatorname{span}\{h, x\}$.

Teоpema 1. Пусть $K \subset X, P_{n} \in \mathfrak{P}_{n}(X, \mathbb{R}) u\left\|P_{n}\right\|_{K} \leqslant 1,\|h\|=1,0<k<n$, $0 \leqslant s \leqslant n-k, h \neq \lambda x$. Справедливы следующие неравенства:

$$
\begin{aligned}
\left|P_{n}^{(k)}(x)\left[h^{k}\right]\right|<\frac{T_{n}^{(k)}\left(\rho_{1}(x, k)\right)}{r_{h}^{k}}, & \rho(x)<\rho_{1}(x, k), \\
\left|P_{n}^{(k+s)}(x)\left[h^{k}, \bar{x}^{s}\right]\right| \leqslant \frac{T_{n}^{(k+s)}\left(\rho_{1}(x, k)\right)}{r_{h}^{k} r_{\bar{x}}^{s}}, & \rho(x)=\rho_{1}(x, k) .
\end{aligned}
$$

Равенство в (9) при $k+s>1$ либо при $k=1, s=0$ и $\rho(x)>\cos (\pi /(2 n))$ имеет место тогда и только тогда, когда для $K, P_{n}, x, h$ выполнен один из трех наборов условий:

$$
\begin{array}{ll}
1^{\circ} & K_{x h}=A_{0}, P_{n}(y)=\gamma\left\{(1-\alpha) T_{n}\left(t_{1}+t_{2}\right)+(-1)^{k} \alpha T_{n}\left(t_{1}-t_{2}\right)\right\}, 0 \leqslant \alpha \leqslant 1,|\gamma|=1 ; \\
2^{\circ} & K_{x h} \backslash A_{0} \neq \varnothing, K_{x h} \subset A_{1}, P_{n}(y)=\gamma T_{n}\left(t_{1}+t_{2}\right),|\gamma|=1 ; \\
3^{\circ} & K_{x h} \backslash A_{0} \neq \varnothing, K_{x h} \subset A_{2}, P_{n}(y)=\gamma T_{n}\left(t_{1}-t_{2}\right),|\gamma|=1 .
\end{array}
$$

Если $k=1, s=0$ и $\rho(x)=\cos (\pi /(2 n))$, то равенство в (9) имеет место тогда и только тогда, когда выполнены следующие условия: 
$4^{\circ}$ либо $K_{x h} \subset A_{1}$, либо $K_{x h} \subset A_{2}$ и $P_{n}\left(x \cos \theta+\sin (\pi /(2 n)) r_{h} h \sin \theta\right)= \pm \sin (n \theta)$.

ЗАмечАниЕ. При $k=n$ неравенство (9) и при $k<n$ и $h=\bar{x}$ неравенства (8) и (9) вытекают из неравенства Шеффера и Даффина и равенство в (9) будет только при $P_{n}(t h)=\gamma T_{n}\left(t / r_{h}\right),|\gamma|=1$.

ДоКАЗАТЕЛЬСТво тЕОРЕмы 1 основано на следующих леммах.

Рассмотрим на плоскости $\mathbb{R}^{2}$ квадрат $K_{2}=\left\{\left(t_{1}, t_{2}\right):\left|t_{1}\right|+\left|t_{2}\right| \leqslant 1\right\}$. Пусть

$$
\bar{T}_{n}\left(t_{1}, t_{2}\right)=\frac{\left\{T_{n}\left(t_{1}+t_{2}\right)+T_{n}\left(t_{1}-t_{2}\right)\right\}}{2}, \quad \widetilde{T}_{n}\left(t_{1}, t_{2}\right)=\frac{\left\{T_{n}\left(t_{1}+t_{2}\right)-T_{n}\left(t_{1}-t_{2}\right)\right\}}{2} .
$$

Пусть $\bar{Q}_{n}$ и $\widetilde{Q}_{n}$ - полиномы из $\mathfrak{P}_{n}\left(\mathbb{R}^{2}, \mathbb{R}\right)$, соответственно четный и нечетный по переменной $t_{2}$.

Лемма 1. Пустъ $k, s \geqslant 0, t^{1}=\left(t_{1}^{1}, t_{2}^{1}\right) \in \mathbb{R}^{2},\left\|\bar{Q}_{n}\right\|_{K_{2}} \leqslant 1,\left\|\widetilde{Q}_{n}\right\|_{K_{2}} \leqslant 1$. Справедливъ следующие неравенства:

$$
\begin{array}{ll}
\left|\bar{Q}_{n_{1}^{s} t_{2}^{k}}^{(s+k)}\left(t_{1}^{1}, t_{2}^{1}\right)\right| \leqslant\left|\bar{T}_{n_{t_{1}^{s} t_{2}^{k}}}^{(s+k)}\left(t_{1}^{1}, t_{2}^{1}\right)\right|, \quad\left|t_{1}^{1}\right| \geqslant 1, \\
\left|\widetilde{Q}_{n_{t_{1}}^{s} t_{2}^{k}}^{(s+k)}\left(t_{1}^{1}, t_{2}^{1}\right)\right| \leqslant\left|\widetilde{T}_{n_{t_{1}^{s} t_{2}^{k}}^{(s+k)}}^{(s+k)}\left(t_{1}^{1}, t_{2}^{1}\right)\right|, \quad\left|t_{1}^{1}\right| \geqslant \cos \frac{\pi}{2 n},
\end{array}
$$

u при $k+s \leqslant n, 2 \leqslant k<n, u k \equiv 0(\bmod 2)$ равенство в (10) имеет место только для $\bar{Q}_{n}= \pm \bar{T}_{n}$, a nрu $k \equiv 1(\bmod 2), 1<k+s \leqslant n, 1 \leqslant k<n$, или при $k=1, s=0$ u $\left|t_{1}^{1}\right|>\cos (\pi /(2 n))$ равенство в (11) имеет место только для $\widetilde{Q}_{n}= \pm \widetilde{T}_{n}$.

Лемма 2. Пусть $Q_{n}=\bar{Q}_{n}+\widetilde{Q}_{n} u\left\|Q_{n}\right\|_{K_{2}} \leqslant 1$. Тогда, если $\bar{Q}_{n}=\bar{T}_{n}$, mо $\widetilde{Q}_{n}=c \widetilde{T}_{n}$, $|c| \leqslant 1 ;$ если $\widetilde{Q}_{n}=\widetilde{T}_{n}$, mo $\bar{Q}_{n}=c \bar{T}_{n},|c| \leqslant 1$.

С использованием теоремы 1 и неравенства Сегё для тригонометрических полиномов доказывается

Tеорема 2. Пустъ $K \subset E^{m}, P_{n} \in \mathfrak{P}_{n}\left(E^{m}, \mathbb{R}\right),\left\|P_{n}\right\|_{K} \leqslant 1,\left\|h_{i}\right\|=1, i=1, \ldots, k$, $n \geqslant k>0$. Тогда

$$
\left|P_{n}^{(k)}\left(x_{0}\right)\left[h_{1}, \ldots, h_{k}\right]\right| \leqslant \frac{T_{n}^{(k)}\left(\rho_{1}\left(x_{0}, k\right)\right)}{r^{k}(K)}
$$

и равенство в (12) имеет место тогда и толъко тогда, когда выполнени следующие условия:

a) $h_{i}=\varepsilon_{i} h, r_{h}=r(K),\left|\varepsilon_{i}\right|=1, i=1, \ldots, k$, если $k=n$, mo $P_{n}(t h)= \pm T_{n}(t / r(K))$, если $k<n$, mо $\rho\left(x_{0}\right)=\rho_{1}\left(x_{0}, k\right)$;

б) если $k<n u r_{x_{0}}=r(K)$, mo $h= \pm \bar{x}_{0}, P_{n}(t h)= \pm T_{n}(t / r(K))$;

в) если $r_{x_{0}}>r(K), n>k>1$ или $n>k=1$ u $\rho\left(x_{0}\right)>\cos (\pi /(2 n))$, mo $K, P_{n}, x_{0}, h$ удовлетворяют условиям пункта $2^{\circ}$ или пункта $3^{\circ}$ теоремы 1 ;

г) если $n>k=1, r_{x_{0}}>r(K)$ u $\rho\left(x_{0}\right)=\cos (\pi /(2 n))$, то $K, P_{n}, x_{0}, h$ удовлетворяют условиям пункта $4^{\circ}$ теоремы 1 и $K_{x_{0} h} \neq A_{0}$.

С использованием формул (5), (6) доказывается

Лемма 3. Пусть $k, s>0, k+s \leqslant n$. Справедливо неравенство

$$
M_{n, k}(t) M_{n-k, s}(t)<M_{n, k+s}(t), \quad t \in(-1,1) .
$$

Из леммы 3 и неравенства Шеффера-Даффина вытекает 
Teоpema 3. Пусть $X=l_{1}^{(m)}, K=\left\{x \in X:\left|x_{i}\right| \leqslant 1, i=1, \ldots, m\right\}, P_{n} \in \mathfrak{P}_{n}(X, \mathbb{R})$, $\left\|P_{n}\right\|_{K} \leqslant 1$. Справедливы следующие неравенства:

$$
\begin{aligned}
& \left\|P_{n}^{(k)}\left(x^{1}\right)\right\|_{\mathscr{L}^{k}}=\max _{\substack{s, k_{i}>0, \sum_{i=1}^{s} k_{i} \leqslant j_{i} \leqslant m \\
1=k}}\left|\frac{\partial^{k} P_{n}\left(x^{1}\right)}{\partial x_{j_{1}}^{k_{1}} \cdots \partial x_{j_{s}}^{k_{s}}}\right| \\
& \leqslant \max _{s, k_{i}>0, \sum_{i=1}^{s} k_{i}=k} N_{n, k_{1}}(\rho) N_{n-k_{1}, k_{2}}(\rho) \cdots N_{n-\sum_{i=1}^{s-1} k_{i}, k_{s}}(\rho), \\
& x^{1} \in K, \quad \rho=\rho\left(x^{1}\right), \\
& \left\|P_{n}^{(k)}\left(x^{1}\right)\right\|_{\mathscr{L}^{k}} \leqslant N_{n, k}\left(\rho\left(x^{1}\right)\right), \quad x^{1} \in X .
\end{aligned}
$$

Пусть $\left|x_{j_{1}}^{1}\right|=\max _{1 \leqslant j \leqslant m}\left|x_{j}^{1}\right|=\rho\left(x^{1}\right)$. Тогда равенство в (13) имеет место, только если $k_{1}=k, P_{n}(x)= \pm T_{n}\left(x_{j_{1}}\right)$, и если $\left|x_{j_{1}}^{1}\right| \leqslant \xi_{1}^{(k)}$, то $x_{j_{1}}^{1}$ является нулем функиии $S_{n}^{(k)}(\cdot)$.

Теорема 4. Пусть $X=\ell_{\infty}^{(2)}$ или $X=\ell_{1}^{(2)} u K=B \subset X$. Для фиксированного $m$ справедливо асимптотическое равенство

$$
\lim _{n \rightarrow \infty} \frac{\max _{\left\|P_{n}\right\|_{K} \leqslant 1}\left\|P_{n}^{(m)}\right\|_{K}}{T_{n}^{(m)}(1)}=1 .
$$

С использованием теоремы 1 и неравенства Гёльдера доказывается

Теорема 5. Пусть $X=\ell_{p}^{(m)}, K=B_{\ell_{q}^{(m)}}, 1 \leqslant p, q \leqslant \infty,\left\|P_{n}\right\|_{K} \leqslant 1$. Тогдa

$$
\left\|P_{n}^{(k)}(x)\right\|_{\mathscr{L}^{k}} \leqslant \min \left(c^{k}, \frac{k^{k}}{k !}\right) \frac{T_{n}^{(k)}\left(\rho_{1}(x, k)\right)}{r^{k}(K)},
$$

где $c=m^{1 / 2-1 / s}$, рри $s=\min (p, q) \geqslant 2, c=m^{1 / u-1 / 2}, n p u u=\max (p, q) \leqslant 2, c=1, n p u$ $1 \leqslant q \leqslant 2 \leqslant p, u$ npu $1 \leqslant p \leqslant 2 \leqslant q$.

B последних двух случалх при $k=n$ оченка (14) точная для любого $x$. Если $1 \leqslant p \leqslant$ $2<q=\infty$, либо $1=q<2 \leqslant p$, то оченка (14) точная только при $\rho(x)=\rho_{1}(x, k)$. Если $1 \leqslant p \leqslant 2 \leqslant q<\infty$ или $1<q \leqslant 2 \leqslant p$, то оченка (14) точнал только при $r_{x}=r(K) u$ $\rho(x)=\rho_{1}(x, k)$.

\section{СПИСОК ЦИТИРОВАННОЙ ЛИТЕРАТУРЫ}

[1] В. И. Скалыга, Изв. РАН. Сер. матем., 69:3 (2005), 179-192. [2] В. А. Марков, О функииях, наименее уклоняющихся от нуля в данном промежутке, Изд-во Императорской Академии наук, СПб, 1892. [3] A.C. Schaeffer, R. J. Duffin, Bull. Amer. Math. Soc., 44:4 (1938), 289-297. [4] В.И. Скалыга, Матем. заметки, 68:1 (2000), 146-150. [5] В.И. Скалыга, Изв. РАН. Сер. матем., 65:6 (2001), 129-172. [6] G. A. Munoz, Y. Sarantopoulos, Math. Proc. Cambridge Philos. Soc., 133 (2002), 515530. [7] Y. Sarantopoulos, Math. Proc. Cambridge Philos. Soc., 110 (1991), 307-312. [8] O. D. Kellogg, Math. Z., 27:1 (1928), 55-64.

В. И. Скалыга

Поступило КБ "Аметист", г. Москва 11.11.2005 